\title{
Network-Based Continuous Space Representation for Describing Pedestrian Movement in High Resolution
}

\author{
Wataru Nakanishi', Takashi Fuse ${ }^{2}$ \\ ${ }^{1}$ Tokyo Institute of Technology \\ 2-12-1-M1-20 Ookayama Meguro, Tokyo, Japan \\ nakanishi@plan.cv.titech.ac.jp \\ ${ }^{2}$ The University of Tokyo \\ 7-3-1 Hongo Bunkyo, Tokyo, Japan \\ fuse@civil.t.u-tokyo.ac.jp
}

\begin{abstract}
A concept of network-based continuous space representation is proposed and applied to the sequential map matching problem with simulation data assuming pedestrian movement. The concept allows for dealing with situations that the resolution of network representation is not high enough to describe the pedestrian movement considering the observation accuracy. The experiment showed that the proposed concept worked well in the example of pedestrian movement along with the sidewalk by estimation of accurate positions.
\end{abstract}

Keywords: road network, offset, continuous space, general state space model, map matching

\section{Introduction}

Map matching is the method to specify the sequence of road network travelled by people by using the sequential measurement of positioning data. A large number of studies on map matching method have been accumulated [1] mainly focusing on vehicle trajectory estimation. Generally, the road network is given in advance as the GIS (Geographic Information System) data (Fig. 1, left), and the positioning data are obtained by GNSS (Global Navigation Satellite System) (Fig. 1, centre). Such methods might be also useful for pedestrian trajectory estimation. For example, estimation of pedestrian behaviour in high-resolution is expected to advance the facility placement along the street. Since devices such as smartphones are moving with individuals while sequentially performing GNSS positioning, expectations for map matching, which is a prerequisite for utilising data obtained there, are increasing.

When capturing the walking trajectory in high resolution, it does not exist directly above the road network. The conventional map matching method, in which measurement coordinates are matched onto the network, has a problem from this viewpoint. In other words, in most methods, the coordinates of the road network as the matching destination are treated as lines having no width and always being on the correct coordinates. This means that the measuring device exists only above the links constituting the network and the map matching problem is corresponding the obtained data including the observation error to the correct position or link of the original network (red line of Fig. 1, right). This is because the application of the conventional map matching is mainly vehicle trajectory and can be regarded that the observation error is much larger than the representation error of the network itself.

However, it may not always hold true in the present and future situation due to following two reasons. First, let us take pedestrian movement as an example and consider a road with a width of tens of meters. Then, on one hand, pedestrians pass through sidewalks on both sides. On the other hand, this road is represented by a single link drawn in the centre. At this time, the observation error about 10 [m] is as large as the error of restoring the coordinates observed by a pedestrian in the sidewalk to the link existing in the centre of the road (pink line of Fig. 1, right). Second, the positioning accuracy will get higher due to the increase of the number of satellites. Then, even if a device is located close to the link, the representation error is still not ignorable. An illustration of this scenario is shown in Fig. 2. Nevertheless, 
considering that behaviour analysis is conducted by describing a route on a network for route choice and traffic assignment problem, trajectory of movement is desired to be associated with a network.

Therefore, in this study, a concept of network-based continuous space representation is proposed to explicitly consider errors of network on continuous space. In this concept, pedestrian positions are represented by the combination of link and offset. This representation has the following two advantages. First, different from the conventional methods, the estimated positions by map matching can be any position, not limited to that just above the road link. Second, the pedestrian trajectory as the matching result is still a sequence of road network, which allows for easy applications for behaviour analyses. The proposed method is applied to the sequential map matching problem with simulation data assuming pedestrian movement.

\section{Continuous Space Representation Using Network}

A Bayesian approach, utilised to cope with uncertainty of observation in the existing studies [2,3], is employed to describe sequential map matching problem. Similar to them, whole model structure is a form of general state space model. Here, a set of latent variables at time instance $t$ is called state vector $\mathbf{x}_{t}$ and a set of observed variables is called observation vector $\mathbf{y}_{t}$. Dynamics of state vector, called system model is a conditional probability $p\left(\mathbf{x}_{t+1} \mid \mathbf{x}_{t}\right)$, and observation process, called observation model is a conditional probability $p\left(\mathbf{y}_{t} \mid \mathbf{x}_{t}\right)$. The core of the proposed concept is the definition of these components (Fig. 3 and 4). Given a road network, any position in continuous space is defined as $\mathbf{x}=(l, d)$, where $l$ indicates a link and $d$ indicates a offset from the corresponding link $l$. Observation is defined as $\mathbf{y}=(x, y, \sigma)$, where $x$ and $y$ are observed coordinates and $\sigma$ is obtained measurement accuracy. Sequential map matching is to estimate the posterior of $\mathbf{x}$ as following,
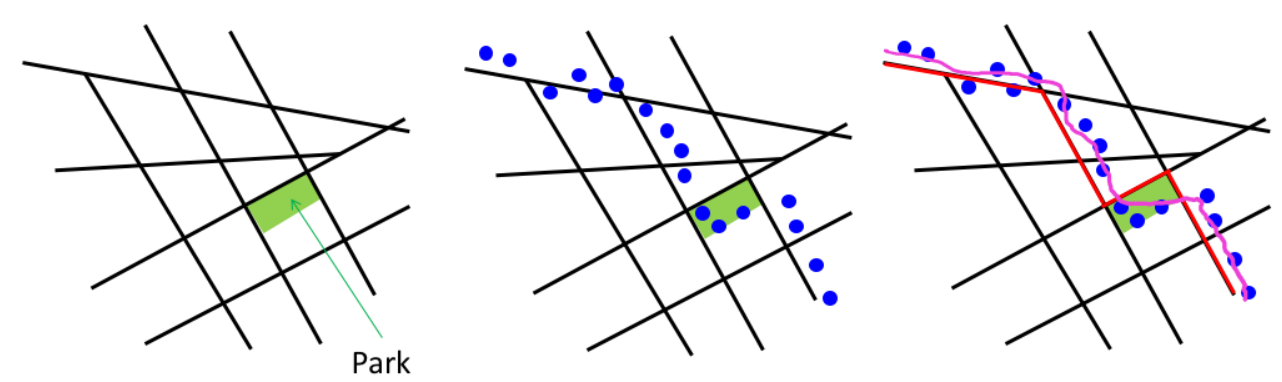

(left) Black lines represent road network (links) with park; (centre) blue dots represent GNSS observations; (right) red lines represent matching result of conventional methods (a sequence of links), and pink lines represent the expected matching results of the proposed method. Walking on sidewalks and across the park can be estimated by this continuous representation.

Fig. 1. Road network, GNSS observation and map matching

\begin{tabular}{l}
\hline sidewalk correct position \\
\hline $\begin{array}{l}\text { representation observed coordinate } \\
\text { error: "offset" }\end{array}$ \\
$\begin{array}{l}\text { link } \\
\text { in the centre } \\
\text { of a road }\end{array}$ \\
\hline
\end{tabular}

Fig. 2. Basic concept for continuous space representation

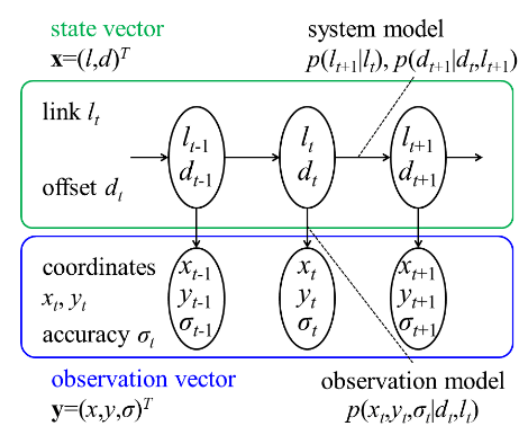

Fig. 3. Space state modelling 


$$
p\left(\mathbf{x}_{t} \mid \mathbf{y}_{1,2, \ldots, t}\right) \propto p\left(\mathbf{y}_{t} \mid \mathbf{x}_{t}\right) p\left(\mathbf{x}_{t} \mid \mathbf{y}_{1,2, \ldots, t-1}\right)=p\left(\mathbf{y}_{t} \mid \mathbf{x}_{t}\right) \int p\left(\mathbf{x}_{t} \mid \mathbf{x}_{t-1}\right) p\left(\mathbf{x}_{t-1} \mid \mathbf{y}_{1,2, \ldots, t-1}\right) d \mathbf{x}_{t-1}
$$

Actual form of state vector is considered as a conditional probability of offset $d$ on link $l$, $p(\mathbf{x})=p(l, d)=p(d \mid l) p(l) \cdot p(d \mid l)$ is defined according to the features of each link (Fig. 5). Then, system model is a combination of $\mathrm{p}\left(l_{t+1} \mid l_{t}\right)$ and $p\left(d_{t+1} \mid d_{t}, l_{t+1}\right)$. The former, link choice model, is treated as Markov transition model. For example, the probability of transition is defined referring to the expected staying time in the same link. The latter, offset dynamics is as follows.

$$
p\left(d_{t+1} \mid d_{t}, l_{t+1}\right) \propto(1-\alpha) p\left(d_{t+1} \mid d_{t}\right)+\alpha p\left(d_{t+1} \mid l_{t+1}\right) \quad \text { if } l_{t+1}=l_{t}, \quad p\left(d_{t+1} \mid d_{t}, l_{t+1}\right)=p\left(d_{t+1} \mid l_{t+1}\right) \quad \text { otherwise }
$$

where $p\left(d_{t+1} \mid d_{t}\right)$ describes a similarity of offset in successive time instances (considered to be random walk), and $\alpha$ is a weight of two components. In addition, observation model at any time instance is as follows (Fig. 4).

$$
p(x, y, \sigma \mid l, d) \propto 1-|\hat{d}-d| / \sigma^{2} \quad \text { if }|\hat{d}-d| \leq \sigma, \quad p(x, y, \sigma \mid l, d)=0 \quad \text { otherwise }
$$

where $p\left(d_{t+1} \mid d_{t}\right)$ describes a similarity of offset in successive time instances (considered to be random walk), and $\alpha$ is a weight of two components. In addition, observation model at any time instance is as follows (Fig. 4). where $\hat{d}$ is the shortest distance between $(x, y)$ and $l$. At last, calculation procedure is explained. Given posterior at time instance $t$, first calculate the predictive distribution of $l_{t+1}$ using Markov transition model. Then, calculate $d_{t+1}$ using equation (2). After obtaining $\mathbf{y}_{t+1}=\left(x_{t+1}, y_{t+1}, \sigma_{t+1}\right)$, calculate equation (3) and then equation (1), the posterior at time instance $t+1$. The estimated distribution of link (the marginal distribution) and the estimated offset distribution given link $l=a$ are as follows.

$$
\begin{aligned}
& p\left(l_{t} \mid \mathbf{y}_{1,2, \ldots, t}\right)=\int p\left(l_{t}, d_{t} \mid \mathbf{y}_{1,2, \ldots, t}\right) d d_{t}=\int p\left(\mathbf{x}_{t} \mid \mathbf{y}_{1,2, \ldots, t}\right) d d_{t} \\
& p\left(d_{t} \mid l_{t}=a, \mathbf{y}_{1,2, \ldots, t}\right)=p\left(d_{t}, l_{t}=a \mid \mathbf{y}_{1,2, \ldots, t}\right) / p\left(l_{t}=a \mid \mathbf{y}_{1,2, \ldots, t}\right)
\end{aligned}
$$

Particle filter $[4,5]$ is employed to calculate the integrals.

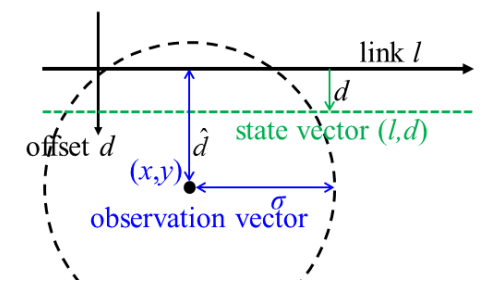

Fig. 4. State and observation vector

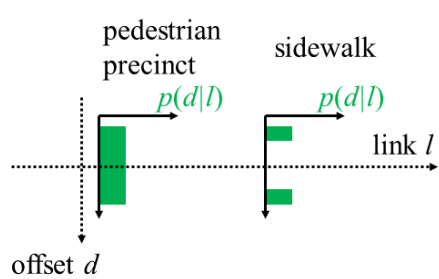

Fig. 5. Various types of $p(d \mid l)$
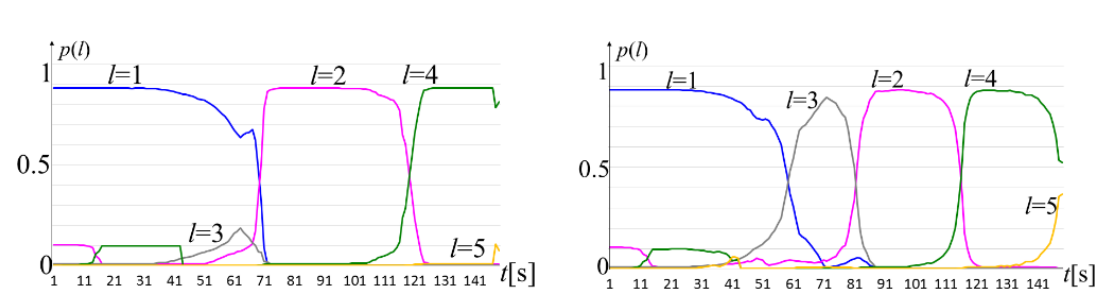
Fig. 7. Estimated link (left: $d \mid l \sim N\left(-10,2^{2}\right)$, right: $d \mid l \sim N\left(0,10^{2}\right)$ )

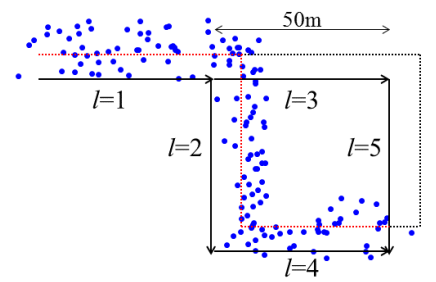

Fig. 6. Virtual network

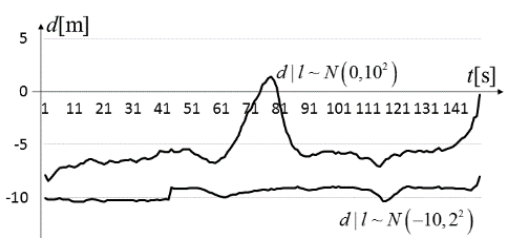

Fig. 8. Estimated offset 


\section{Application to Simulation Data}

The proposed method is applied to simulation data on several virtual network with several parameter settings. Overall, the proposed method works well in both estimating the correct link and the amount of offset. One example of network is shown in Fig. 6. Road network is represented by black solid lines and the length of each link is $50[\mathrm{~m}]$. However, pedestrian actually moves along sidewalks (red dashed lines), which are located at $d=-10$ of each link. Observation coordinates are generated by a pedestrian moves $1[\mathrm{~m} / \mathrm{s}]$ along the link 1,2 and 4 in this order with accuracy of $\sigma=5[\mathrm{~m}]$. In this way, a set of observation is obtained from $t=1$ to 150 (shown by blue dots in Fig. 7). Two distributions of $p(d \mid l)$, the component of state vector, are assumed as $d \mid l \sim N\left(-10,2^{2}\right)$ and $d \mid l \sim N\left(0,10^{2}\right)$. The former corresponds the situation that the information of sidewalk is included and the latter the conventional method. In this setting, the estimated results are shown in Fig. 7 and 8. The former distribution is superior in both estimating link and offset; in the latter setting, the wrong $\operatorname{link} l=3$ and offset about $d=0$ are estimated around $t=70$.

\section{Conclusion}

The sequential map matching with network-based continuous space representation was proposed and applied to simulation data assuming pedestrian movement. The experiment showed that the proposed method worked well in the example of pedestrian movement along with the sidewalk.

\section{Acknowledgements}

This work was supported by JSPS KAKENHI Grant Number JP15K18131.

\section{References}

[1] M. A. Quddus, W. Y. Ochieng, and R. B. Noland, "Current map-matching algorithms for transport applications: State-of-the art and future research directions," Transp. Res. Part C, Vol.15, no.5, pp.312-328, 2007.

[2] W. Kim, G. Jee and J. Lee, "Efficient use of digital road map in various positioning for ITS," Proc. of IEEE Symposium on Position Location and Navigation, 2000.

[3] T. Hunter, R. Herring, P. Abbeel and A. Bayen, "Path and travel time inference from GPS probe vehicle data," Proc. of the Neural Information Processing Systems Foundation (NIPS), 2009.

[4] N. Gordon, D. Salmond, and A. Smith, "Novel approach to nonlinear / non-Gaussian Bayesian state estimation," Radar and Signal Processing, IEE Proc. F, Vol.140, no.2, pp.107-113, 1993.

[5] G. Kitagawa, "Monte Carlo filter and smoother for non-Gaussian nonlinear state space models," Journal of Computational and Graphical Statistics, Vol.5, no.1, pp.1-25, 1996. 